Bulletin of Mathematical Biology (2002) 64, 673-702

doi:10.1006/bulm.2002.0293

Available online at http://www.idealibrary.com on IDE $\mathbf{A}^{[}$

\title{
Mathematical Modelling of Flow Through Vascular Networks: Implications for Tumour-induced Angiogenesis and Chemotherapy Strategies
}

\author{
S. R. McDOUGALL \\ Department of Petroleum Engineering, \\ Heriot-Watt University, \\ Edinburgh EH14 4AS, \\ U.K.
}

\author{
A. R. A. ANDERSON AND M. A. J. CHAPLAIN \\ Department of Mathematics, \\ The SIMBIOS Centre, University of Dundee, \\ Dundee DD1 4HN, \\ U.K.
}

\author{
J. A. SHERRATT \\ Department of Mathematics, \\ Heriot-Watt University, \\ Edinburgh EH14 4AS, \\ U.K.
}

Angiogenesis, the formation of blood vessels from a pre-existing vasculature, is a process whereby capillary sprouts are formed in response to externally supplied chemical stimuli. The sprouts then grow and develop, driven initially by endothelial cell migration, and organize themselves into a branched, connected network structure. Subsequent cell proliferation near the sprout-tip permits further extension of the capillary and ultimately completes the process. Angiogenesis occurs during embryogenesis, wound healing, arthritis and during the growth of solid tumours. In this paper we initially generate theoretical capillary networks (which are morphologically similar to those networks observed in vivo) using the discrete mathematical model of Anderson and Chaplain. This discrete model describes the formation of a capillary sprout network via endothelial cell migratory and proliferative responses to external chemical stimuli (tumour angiogenic factors, TAF) supplied by a nearby solid tumour, and also the endothelial cell interactions with the extracellular matrix.

The main aim of this paper is to extend this work to examine fluid flow through these theoretical network structures. In order to achieve this we make use of flow modelling tools and techniques (specifically, flow through interconnected networks) from the field of petroleum engineering. Having modelled the flow of a basic fluid through our network, we then examine the effects of fluid viscosity, blood vessel size (i.e., diameter of the capillaries), and network structure/geometry, upon: (i) the rate of flow through the network; (ii) the amount of fluid present in the complete network at any one time; and (iii) the amount of fluid reaching the tumour. The incorporation of fluid flow through the generated vascular networks 
has highlighted issues that may have major implications for the study of nutrient supply to the tumour (blood/oxygen supply) and, more importantly, for the delivery of chemotherapeutic drugs to the tumour. Indeed, there are also implications for the delivery of anti-angiogenesis drugs to the network itself. Results clearly highlight the important roles played by the structure and morphology of the network, which is, in turn, linked to the size and geometry of the nearby tumour. The connectedness of the network, as measured by the number of loops formed in the network (the anastomosis density), is also found to be of primary significance. Moreover, under certain conditions, the results of our flow simulations show that an injected chemotherapy drug may bypass the tumour altogether.

(c) 2002 Society for Mathematical Biology. Published by Elsevier Science Ltd. All rights reserved.

\section{INTRODUCTION}

Angiogenesis (syn neovascularization), the formation of blood vessels from a pre-existing vasculature, is a crucial component of many mammalian growth processes. It occurs in early embryogenesis during the formation of the placenta, after implantation of the blastocyst in the uterine wall (Graham and Lala, 1992). It also occurs, in a controlled manner, in adult mammals during tissue-repair (Arnold and West, 1991; Pettet et al., 1996). By contrast, uncontrolled or excessive blood-vessel formation is essential for tumourigenesis and is also observed in arthritis, abnormal neovascularization of the eye, duodenal ulcers, and following myocardial infarction (Folkman and Klagsbrun, 1987; Folkman, 1985, 1995). These instances may be considered pathological examples of angiogenesis (Muthukkaruppan et al., 1982; Folkman and Brem, 1992). In each case, however, the well-ordered sequence of events characterizing angiogenesis is the same, beginning with the rearrangement and migration of endothelial cells from a pre-existing vasculature and culminating in the formation of an extensive network, or bed, of new capillaries (Madri and Pratt, 1986). In this paper we focus exclusively on tumour-induced angiogenesis, although the results from the mathematical model and flow simulations have implications for neovascularization arising in the other growth processes mentioned earlier.

The first event of tumour-induced angiogenesis involves the cancerous cells of a solid tumour secreting a number of chemicals, collectively known as tumour angiogenic factors, or TAF (Folkman and Klagsbrun, 1987), into the surrounding tissue. These factors diffuse through the tissue space creating a chemical gradient between the tumour and any existing vasculature. Upon reaching any neighbouring blood vessels, endothelial cells lining these vessels are first induced to degrade the parent vessel basement membranes and then migrate through the disrupted membrane towards the tumour.

The initial response of the endothelial cells to these angiogenic factors is a chemotactic one (Sholley et al., 1984; Terranova et al., 1985; Paweletz and Knierim, 1989; Stokes et al., 1990), initiating the migration of the cells towards the tumour. 
Following this, small, finger-like capillary sprouts are formed by accumulation of endothelial cells, which are recruited from the parent vessel. The sprouts grow in length due to the migration and further recruitment of endothelial cells (Cliff, 1963; Schoefl, 1963; Warren, 1966; Ausprunk and Folkman, 1977; Sholley et al., 1984) and continue to move toward the tumour directed by the motion of the leading endothelial cell at the sprout-tip. Further sprout extension occurs when some of the endothelial cells of the sprout-wall begin to proliferate. Cell division is largely confined to a region just behind the cluster of mitotically inactive endothelial cells that constitute the sprout-tip. This process of sprout-tip migration and proliferation of sprout-wall cells forms solid strands of endothelial cells amongst the extracellular matrix. The cells continue to make their way through the extracellular matrix which consists of interstitial tissue, collagen fibre, fibronectin and other components (Liotta et al., 1983; Paweletz and Knierim, 1989). Interactions between the endothelial cells and the extracellular matrix are very important and directly affect cell migration. In particular, the specific interactions between the endothelial cells and fibronectin, a major component of the extracellular matrix, have been shown to enhance cell adhesion to the matrix. Consequently, in addition to the chemotactic response of the endothelial cells to the TAF, there is a complementary haptotactic response to the fibronectin present within the extracellular matrix (Bowersox and Sorgente, 1982).

Initially, the sprouts arising from the parent vessel grow in an essentially parallel manner. However, once the finger-like capillary sprouts have reached a certain distance from the parent vessel, they are seen to incline toward each other (Paweletz and Knierim, 1989), leading to numerous tip-to-tip and tip-to-sprout fusions known as anastomoses. Such anastomoses result in the fusing of the finger-like sprouts into a network of poorly perfused loops or arcades. Following this process of anastomosis, the first signs of circulation can be recognized and from the primary loops, new buds and sprouts emerge repeating the angiogenic sequence of events and providing for the further extension of the new capillary bed. The production of new capillary sprouts from the sprout-tips is often referred to as sprout branching, and as the sprouts approach the tumour, their branching dramatically increases until the tumour is eventually penetrated, resulting in vascularization (Muthukkaruppan et al., 1982; Itoh et al., 2000).

During the vascular phase of growth, the tumour is highly regulated by the capillary network around it. Nutrients are supplied to the tumour via this network, and it provides the initial route for invading cancer cells to escape from the primary tumour and form metastases. Moreover, it is through the vascular network that chemotherapeutic drugs will be delivered to the tumour. All of these processes depend crucially on the blood flow within the vascular network, which is therefore a central regulator of the vascular phase of tumour development. Although much is known about the physical structure of the capillary network, which has been studied in detail both experimentally [see, for example, Paweletz and Knierim (1989), for a review] and theoretically [see, for example, Anderson and Chaplain (1998), 
and references therein], attempts to study the blood flow within the vasculature surrounding solid tumours (and also, by implication, the drug supply to the tumours) are relatively few. Consequently, the main focus of this paper is to address the topic of modelling flow through tumour-induced capillary networks.

Tumour-induced angiogenesis provides the crucial link between the avascular phase of solid tumour growth and the more harmful vascular phase, wherein the tumour invades the surrounding host tissue and blood system (Chaplain, 1996). However, these apparently insidious features of tumour-induced angiogenesis are now being used in new treatments of cancer and the clinical importance of angiogenesis as a prognostic tool is now well-recognised (Harris et al., 1994; Bikfalvi, 1995; Ellis and Fidler, 1995; Gasparini, 1995; Gasparini and Harris, 1995; Norton, 1995). Anti-angiogenesis strategies are also being developed as potentially powerful, noninvasive weapons against the spread of cancer (Herblin and Gross, 1994; Harris et al., 1996; Harris, 1997).

Partial differential equation modelling was first applied to tumour-induced angiogenesis by Balding and McElwain (1985) and then developed by Chaplain and Sleeman (1990), who studied concentration profiles of tumour angiogenic factors (TAFs), and Chaplain and Stuart (1993). More recent work has shown that the structure and morphology of the capillaries and the network also depend crucially on the interactions between the endothelial cells and local gradients of extracellular matrix components (Orme and Chaplain, 1996; Anderson and Chaplain, 1998; Holmes and Sleeman, 2000; Levine et al., 2001). Although these continuum models are capable of capturing certain important features of angiogenesis, such as average sprout density, average vessel growth rates and network expansion rates, they are limited because they cannot reproduce the morphology of branched, connected networks at the level of detail required for the flow simulations.

In order to capture the fine-scale detail of these inter-connected networks, it is necessary to use some form of discrete modelling technique i.e., one that operates at the level of single endothelial cells. There have been several approaches using spatially discrete representations e.g., Stokes and Lauffenburger (1991), Nekka et al. (1996), Anderson and Chaplain (1998) and Gödde and Kurz (2001). In this paper we will utilize the approach of Anderson and Chaplain (1998) in order to generate our theoretical capillary networks, and details of the model are given in Section 2.1 [see also Anderson et al. (1997)]. The main advantage of using this technique is the ability to follow the motion of individual endothelial cells at the capillary tips (where migration occurs) and to enable one to include important processes at the individual cell level such as proliferation, branching and loop formation. The mathematical model focussed on three key variables involved in tumour-induced angiogenesis; namely, endothelial cells, TAFs and fibronectin, each of which has a crucial role to play. The principal aim of this paper is to extend the work of Anderson and Chaplain (1998) by incorporating flow through the network (e.g., blood, chemotherapy drugs) and to consider the implications for tumour growth and chemotherapy treatment strategies. 
The layout of the remainder of the paper is as follows. Before modelling flow through the capillary networks, we first briefly summarize the model of Anderson and Chaplain (1998), which we will use to generate our theoretical network structures. In the first instance, using conservation laws and a continuum approach, we will derive a system of coupled nonlinear partial differential equations modelling the initial migratory (chemokinetic/chemotactic) response of the endothelial cells to TAF, production of fibronectin and degradation of matrix components (fibronectin), and the haptotactic response of the endothelial cells to components of the extracellular matrix (specifically fibronectin). From a discretized form of these partial differential equations, a discrete (biased random walk) model will be derived enabling the paths of individual endothelial cells located at the sprout-tips, and hence the individual capillary sprouts, to be followed. The processes of sprout branching, anastomosis (loop formation) and cell proliferation will be incorporated in this discrete biased random walk model. Having generated realistic capillary network structures, we will then turn our attention to the main focus of the paper-to examine the flow of various fluids (blood, drugs) through these structures and consider the implications for nutrient delivery to tumours and chemotherapy strategies.

\section{The Mathematical Model of Network Growth}

In this section we give a brief description of the mathematical model of Anderson and Chaplain (1998). This is based upon the experimental system of Muthukkaruppan et al. (1982), whereby a small solid tumour or fragment of tumour was implanted in the cornea of a test animal close to the limbal vessels of the eye-these vessels are lined with endothelial cells. We denote the endothelial cell density per unit area by $n$, the TAF concentration by $c$ and the fibronectin concentration by $f$. For the migration of the endothelial cells we assume: (i) that there is a small amount of random motion (which may be dependent on the underlying TAF concentration i.e., chemokinesis); (ii) that cells respond chemotactically to gradients of TAF; and (iii) that cells respond haptotactically to gradients of fibronectin in the matrix. Proliferation of the cells is incorporated at the individual level via the discrete model described in the next section. For the TAF, we assume that a (quasi) steady state distribution already exists in the matrix (the TAF having initially been secreted by the tumour cells) thereby providing an initial concentration gradient to which the endothelial cells can respond. As the endothelial cells migrate through the tissue, there is some binding of the TAF to the cells and we model this with a simple uptake function $-\eta n c$. Fibronectin exists in the matrix in bound form and therefore there is no diffusion term in the fibronectin equation. Endothelial cells are known to produce fibronectin as they migrate and we assume this is at a rate $\beta n$. The endothelial cells also degrade the matrix as they migrate and this is brought about by the cells secreting matrix-degrading enzymes such as matrix-metallo-proteases (MMPs) and urokinase plasminogen 
activator (uPA). The complete degradation process is rather complicated involving bound and unbound, active and inactive forms of the enzymes. However, the degradation is a tightly regulated and highly localized process restricted to the immediate pericellular environment (Paweletz and Knierim, 1989; Matrisian, 1992; Alberts et al., 1994; Andreasen et al., 1997; Pepper, 2001) and therefore we make the simplifying assumption that the endothelial cells degrade the matrix (fibronectin) upon contact. We include a simple degradation term, $-\gamma n f$, in the fibronectin equation to reflect this fact [a slightly more detailed treatment of modelling the action of matrix degrading enzymes may be found in Perumpanani et al. (1996); Anderson et al. (2000); Levine et al. (2001)]. With these modelling assumptions the full (nondimensional) system of equations becomes:

$$
\begin{aligned}
& \frac{\partial n}{\partial t}=\overbrace{D \nabla \cdot(d(c) \nabla n)}^{\text {chemokinesis }}-\overbrace{\chi \nabla \cdot(g(c) n \nabla c)}^{\text {chemotaxis }}-\overbrace{\rho \nabla \cdot(n \nabla f)}^{\text {haptotaxis }}, \\
& \frac{\partial c}{\partial t}=-\overbrace{\eta n c}^{\text {uptake }}, \\
& \frac{\partial f}{\partial t}=\overbrace{\beta n}^{\text {synthesis }}-\overbrace{\gamma n f}^{\text {degradation }} .
\end{aligned}
$$

The (nondimensional) equations are taken to hold on a two-dimensional (2D) domain, the unit square $[0,1] \times[0,1]$, with no-flux boundary conditions. Appropriate initial conditions are imposed for $n, c$, and $f$. All parameter values have been estimated, as far as possible, from available experimental data (Anderson and Chaplain, 1998) with the unit of length taken to be $2.5 \mathrm{~mm}$ and the unit of time taken to be 1.5 days. The key (nondimensional) motility parameters have been estimated as $D=0.00035, \chi=0.38$ and $\rho=0.34$. Full details of the initial conditions used, the nondimensionalization process, and references to the experimental results can be found in Anderson and Chaplain (1998). In the simulations carried out in the following sections we took as a chemotaxis function $g(c)=1 /(1+c)$. Finally we took the chemokinesis function $d(c)$ to be a constant [i.e., $d(c)=1$, simple cell random motility, with coefficient $D$ ]. This is in line with recent work by Schor et al. (2002) using a three-dimensional (3D) collagen gel cell migration assay (a sandwich assay) and the well-known cytokine TGF- $\beta$. Results here show that although all three isoforms of TGF- $\beta$ (i.e., TGF- $\beta 1$, TGF- $\beta 2$ and TGF- $\beta 3$ ) do indeed stimulate fibroblast migration and endothelial cell migration in the $3 \mathrm{D}$ collagen gel in a chemokinetic manner, the actual quantitative effect on cell migration over and above that observed in the control experiments is minimal. The data show that constant random motility of the cells is an excellent approximation over four orders of magnitude of cytokine concentration. We note that previous cell migration experiments carried out in a 3D collagen gel (Schor et al., 1999) with a different cytokine (platelet derived growth factor, PDGF) and fibroblasts 


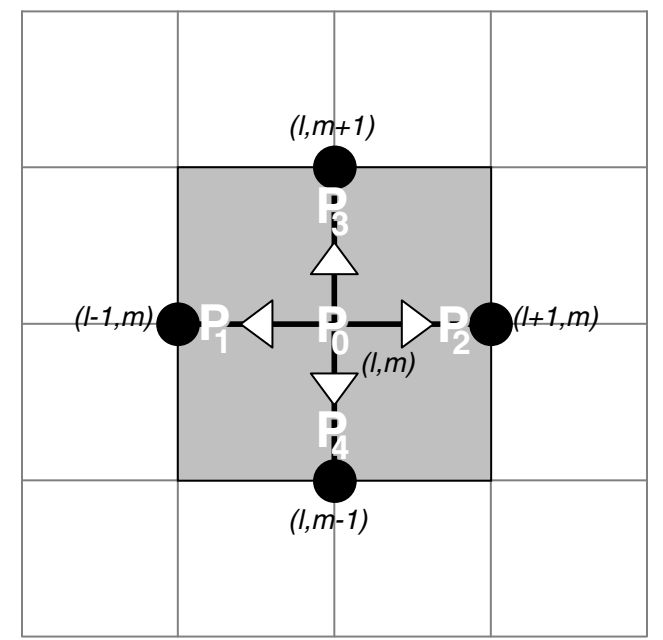

Figure 1. Movement probabilities for an individual endothelial cell generated from the model of Anderson and Chaplain (see text for details). The current endothelial cell is located at position $(l, m)$ and has the chance to move to one of the four orthogonal neighbours (filled circles) with a probability of $P_{1}, P_{2}, P_{3}, P_{4}$ or remain stationary with a probability $P_{0}$.

show that PDGF does elicit a larger chemokinetic response in the cells. However we estimate that the maximum effect of this is to increase the parameter $D 10$-fold to around 0.003 which still means that the taxis components in the cell migration dominate [simulations performed using this value of $D$ in the discrete model generated capillary networks that were very similar in structure to those shown in Fig. 2(a) and (b)].

The results of the numerical simulations of the above system showed interesting spatio-temporal dynamics and demonstrated two important aspects of capillary network formation: (i) in agreement with previous models, (Stokes and Lauffenburger, 1991; Chaplain and Stuart, 1993) a sufficiently strong chemotactic response is necessary for the initial outgrowth of the capillary network; (ii) interactions between endothelial cells and the extracellular matrix are very important for the successful development of the network. Full details can be found in Anderson and Chaplain (1998). In summary, the continuum model captured the important (large-scale) qualitative features of capillary network growth. However, important processes on a smaller scale, such as sprout branching, localized endothelial cell proliferation, are not captured. In order to achieve this (and to proceed with the subsequent flow simulation) we require to develop a model applicable at the level of a single endothelial cell. This may be carried out by a number of different techniques (e.g., stochastic differential equations, cellular automata, diffusion limited aggregation). However we follow the method developed by Anderson and Chaplain (1998) and this is explained fully in the next section. 


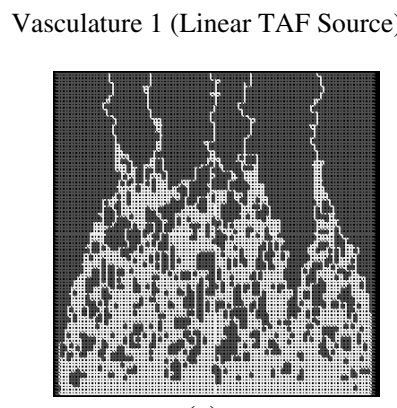

(a)

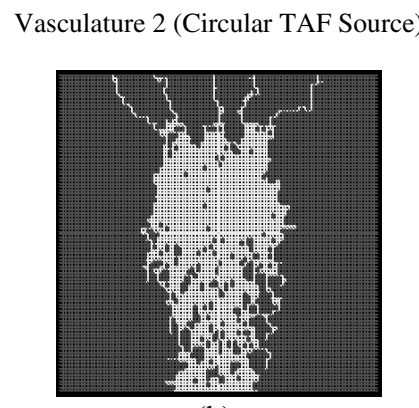

(b)

Figure 2. Vascular networks generated by the model of Anderson and Chaplain (see text for details). In (a) the vasculature is responding to a linear source of tumour cells placed along the lower (horizontal) boundary, while in (b) the response is to a small circular tumour located midway along the lower boundary. In both cases the parent vessel lies along the upper (horizontal) boundary. These vasculatures are then used to generate the flow networks used in the flow simulations (see Figs 3 and 4 for details).

2.1. The discrete mathematical model. Having defined the continuum model via system (1) of partial differential equations (PDEs), we now formulate the discrete model which involves using a discretized form of the PDEs. Essentially we generate a biased random walk model which governs the movement of individual endothelial cells at the capillary sprout tips. We assume that all cells within the capillary sprout are contiguous (Paweletz and Knierim, 1989) and therefore tracking the path of an individual tip cell defines the shape of the whole sprout. We first discretize (1) using the standard Euler finite difference approximation (Mitchell and Griffiths, 1980). This involves approximating the continuous 2D domain $[0,1] \times[0,1]$ in the usual way as a grid of discrete points (mesh size $h$ ), and time $(t)$ by discrete increments (magnitude $k$ ). The full discretized system and all other related details are given in Anderson and Chaplain (1998). For clarity of exposition, we present the form of the discrete endothelial cell equation:

$$
n_{l, m}^{q+1}=n_{l, m}^{q} P_{0}+n_{l+1, m}^{q} P_{1}+n_{l-1, m}^{q} P_{2}+n_{l, m+1}^{q} P_{3}+n_{l, m-1}^{q} P_{4}
$$

where the subscripts specify the location on the grid and the superscripts the time steps. That is $x=l h, y=m h$ and $t=q k$ where $l, m, k, q$ and $h$ are positive parameters.

For our discrete model, we use the five coefficients $P_{0}$ to $P_{4}$ from (2) to generate the motion of an individual endothelial cell. These coefficients can be thought of as being proportional to the probabilities of the endothelial cell being stationary $\left(P_{0}\right)$ or moving left $\left(P_{1}\right)$, right $\left(P_{2}\right)$, up $\left(P_{3}\right)$ or down $\left(P_{4}\right)$ (see Fig. 1 for a schematic diagram). The exact forms of $P_{0}$ to $P_{4}$ involve functions of the fibronectin and TAF concentrations in a local neighbourhood of the individual endothelial cell i.e., the motion of individual cells is governed by the local interactions between the cells and their surrounding milieu. Once again full details can be found in Anderson and Chaplain (1998). 


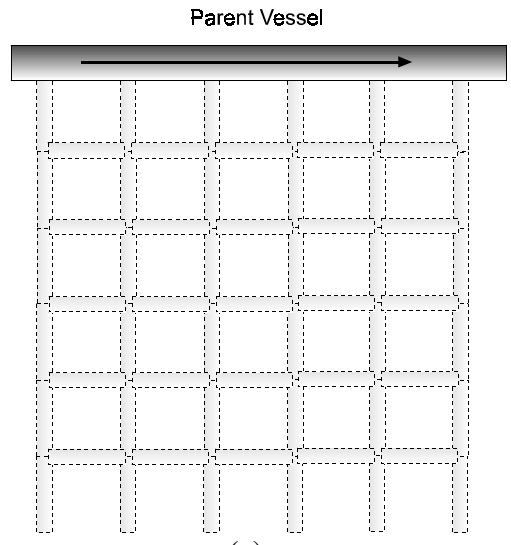

(a)

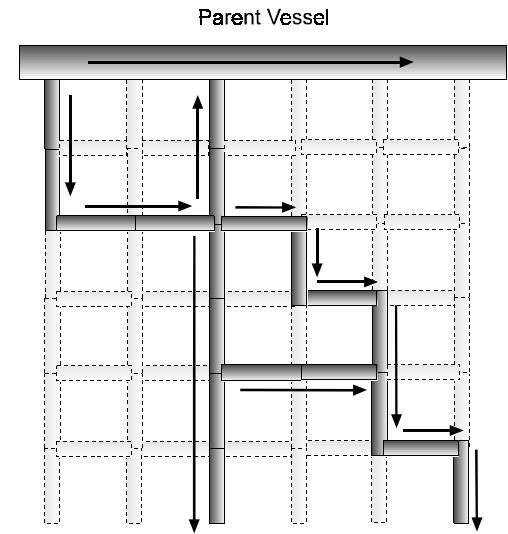

(b)

Figure 3. Schematic diagram showing construction of the vascular network; (a) the lattice template onto which the vascular pattern generated by the discrete mathematical model is mapped, (b) schematic section of the mapped vasculature showing how blood flows in, through and out of the network along the various network bond elements (arrows indicate flow direction).

Before proceeding to the simulation section, where we describe how we generate the capillary networks, first we briefly discuss the manner in which we explicitly incorporate the processes of branching, anastomosis and cell proliferation into the discrete model.

2.2. Rules for branching, anastomosis and cell proliferation. We will assume that the generation of new sprouts (branching) occurs only from existing sprout-tips and that the newly formed sprouts are unlikely to branch immediately. We assume that each sprout-tip has a probability, $P_{b}$, of generating a new sprout (branching) and that this probability is dependent on the local TAF concentration. The simple rule used is that as the TAF concentration increases the probability of generating new sprouts (branching) increases.

Anastomosis, the formation of loops by capillary sprouts, is another very important feature of angiogenesis, which can be captured explicitly by the discrete model. During capillary sprout growth, it is observed that neighbouring sprouts come near to each other at their leading tips and then may fuse together to develop loops. Tip cells are also seen to fuse with the sides of capillary sprouts (Paweletz and Knierim, 1989). In our discrete model as the sprouts progress towards the tumour, driven by the movement probabilities of (2) at each time step of the simulation, the endothelial cells at the sprout-tips can move to any of the four orthogonal neighbours on the discrete grid. If upon one of these moves another sprout is encountered, then anastomosis can occur and a loop is formed. We assume that as a result of a tip-to-tip anastomosis, one of the original sprouts continues to grow (the choice of which is purely random) and the other fuses to form the loop (Paweletz and Knierim, 1989). 


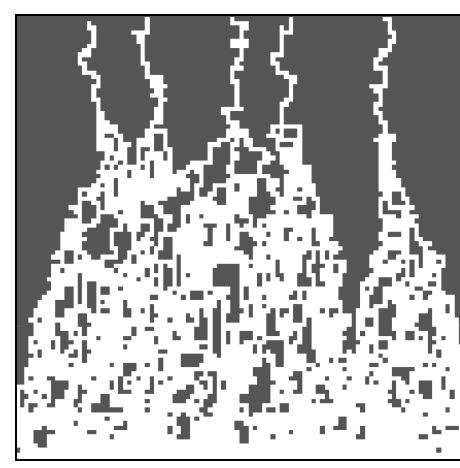

(a)

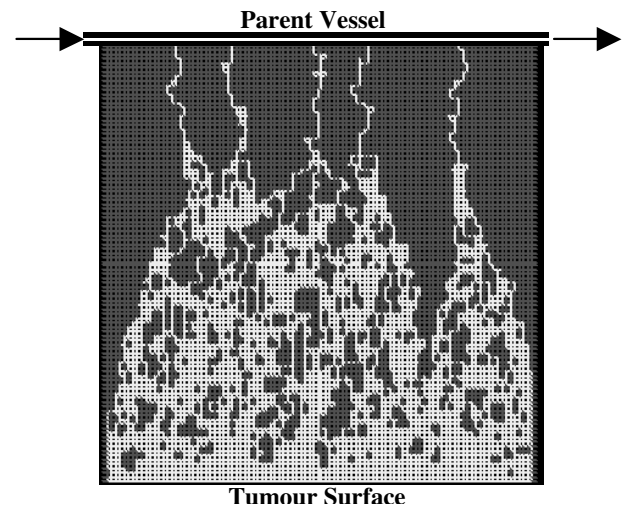

(b)

Figure 4. Illustration of how the mapping technique is used to construct a vascular flow network: (a) shows a theoretical network generated by the discrete mathematical model, while (b) shows the corresponding flow network.

Endothelial cell doubling time has been estimated at $18 \mathrm{~h}$ (Williams, 1987) and we model the process of cell division in the discrete model by assuming that some of the cells behind the sprout-tip divide (into two daughter cells) every $18 \mathrm{~h}$. We assume that this has the effect of increasing the length of a sprout by approximately one cell length every $18 \mathrm{~h}$. Owing to the inherent randomness of the discrete model, proliferation will occur asynchronously in separate sprouts, as is observed experimentally (Paweletz and Knierim, 1989).

2.3. Simulation process for the discrete model. Each time step of the simulation process involves solving the discrete form of system (1) numerically to generate the five coefficients $P_{0}$ to $P_{4}$. Probability ranges are then computed by summing the coefficients to produce five ranges, $R_{0}=0$ to $P_{0}$ and

$$
R_{j}=\sum_{i=0}^{j-1} P_{i} \quad \text { to } \quad \sum_{i=0}^{j} P_{i} \quad \text { where } \quad j=1-4 .
$$

We then generate a random number between 0 and 1 and, depending upon the range in which this number falls, the individual endothelial cell under consideration will either remain stationary $\left(R_{0}\right)$ or move left $\left(R_{1}\right)$, right $\left(R_{2}\right)$, up $\left(R_{3}\right)$ or down $\left(R_{4}\right)$. The larger a particular range, the greater the probability that the corresponding coefficient will be selected. Each endothelial cell is therefore restricted to move to one of its four orthogonal neighbouring grid points or remain stationary at each time step.

Since the main focus of the current study is the incorporation of blood flow through vascular networks, use of the discrete model was restricted to the generation of two structurally different capillary networks. The first network was generated in response to a linear concentration profile of TAFs arising from a line source 
of tumour cells or, equivalently a large solid tumour [see Fig. 2(a)]. The second network was generated in response to a radially symmetric concentration profile of TAF arising from a small circular tumour [see Fig. 2(b)]. Both simulations were carried out on a $100 \times 100$ grid which is a discretization of the unit square $[0,1] \times[0,1]$ [this is equivalent to a dimensional domain of length $2.5 \mathrm{~mm}$, the average distance of a tumour implant from the limbal vessels in the corneal experiments of Muthukkaruppan et al. (1982)], with a space step of $h=0.01$ (equivalent to a dimensional distance of $25 \mu \mathrm{m}$ ). No flux boundary conditions were imposed on the square grid, restricting the endothelial cells to within the grid.

It is evident from Fig. 2 that different source (tumour) geometries lead to vastly different vascular structures and network connectedness (anastomoses density). We will find that such differences play a crucial role in determining the efficacy of drug delivery to the tumour once flow has been incorporated into the model. In the following section we will describe how we incorporate flow through a capillary network which has been generated from our discrete mathematical model.

\section{Modelling Flow Through Vascular Networks}

In this section we focus on the main aim of this paper, which is to model and examine flow through vascular networks surrounding solid tumours. Examination of any photomicrograph relating to vascularization immediately demonstrates why the modelling of fluid flow through a vascular network is such a challenging task. Fluid mechanical issues notwithstanding, the underlying network topology is itself rather complex, consisting of tortuous interconnected blood vessels embedded within a host tissue. Whilst there appear to have been comparatively few attempts to model flow through realistic vascular structures (reviewed later), a large amount of related research has been carried out in the (seemingly unconnected) field of petroleum engineering. As its etymology suggests, petroleum is usually found trapped within the interstices of a rock, and as the rock structures themselves generally consist of interconnected pore ensembles (cf. capillaries) set within a solid matrix (cf. host tissue), the link between hydrocarbon flow through pore systems and flow through vascular networks becomes clear.

A fully interconnected network was first used by Fatt (1956) in the context of oil and water flow through reservoir rock and, although the 2D lattice used was extremely small (200-400 elements), the novelty of the approach encouraged great interest in the subject. Improvements on this early model were soon forthcoming (Rose, 1957; Dodd and Kiel, 1959) and a substantial literature relating to pore-scale modelling now exists [see Dullien (1992), for an overview]. Indeed, the recent advent of high speed computing has lead to the design and implementation of systems containing hundreds of thousands of pore elements and facilitated the production of statistically meaningful 3D simulations (McDougall and Sorbie, 1997). 
Related research in the field of microcirculatory blood perfusion has only emerged relatively recently [see Schmid-Schonbein (1999), for a review] and modelling attempts tend to fall into two broad categories: biomechanical properties of blood/blood vessels, and flow-induced evolution of microvascular networks. Studies associated with the former include; nonNewtonian effects and haematocrit correlations (Sutton and Schmid-Schonbein, 1994), application of Reynolds lubrication theory to blood flow through nonuniform capillaries (Secomb and Hsu, 1996), and modelling of viscoelastic vessel compliance (Price and Skalak, 1995). Studies of flow in microvascular networks have focussed primarily upon structural adaptations of arterial and venous trees (Honda and Yoshizato, 1997; Pries et al., 1998; Gödde and Kurz, 2001), and haematocrit evolution during network perfusion (Schmid-Schonbein et al., 1980; Levin et al., 1986; Pries et al., 1990). More recently, studies dealing with blood perfusion within tumours have been reported (Baish et al., 1996, 1997). The first of these papers utilized an invasion percolation model (Wilkinson and Willemsen, 1983) to construct a small $(32 \times 32)$ artificial 2D network that had the same fractal dimension as a vasculature observed in a murine dorsal skinfold chamber preparation. The model was used to help explain why tumour vascular resistance is found to be higher than that observed in normal vasculature; tumour oxygenation was also examined. The second paper in the series took a less sophisticated approach to examine transmural coupling in tumours-a regular square network and a pair of countercurrent cylinders were used to model various aspects of fluid exchange between the microcirculation and tumour interstitium.

In this paper, we examine a rather different aspect of the microcirculationwe attempt to study blood flow within the vasculature surrounding solid tumours (and also, by implication, the drug supply to the tumours). Moreover, in contrast to some other approaches, we generate vascular networks using models that are firmly underpinned by the governing biological processes (chemokinesis, chemotaxis, and haptotaxis)—-hence our flow networks are firmly grounded in physical reality.

3.1. Network model design. In its most general form, a network flow model may be considered in three space dimensions (indeed, such networks are regularly used in the petroleum engineering context). However, in order to be consistent with the theoretical networks generated via the discrete model of the previous section [Fig. 2(a) and (b)], the flow simulations reported here are restricted to 2D lattice networks of bond elements - these can be thought of as being straight, rigid cylindrical capillaries that join adjacent nodes (junctions), although the constraints of rigidity and cylindrical geometry can easily be relaxed (Fig. 3). Each capillary element forming part of the microvasculature is assigned a radius drawn from an input probability distribution function (PDF). Now, for a single capillary element (joining nodes $i$ and $j$ ) of radius $R_{i j}$ and length $L_{i j}$, the elemental flow rate in the 
capillary is assumed to follow Poiseuille's law:

$$
Q_{i j}=\frac{\pi R_{i j}^{4} \Delta P_{i j}}{8 \mu L_{i j}}
$$

where $\mu$ is the fluid viscosity and $\Delta P_{i j}=\left(P_{i}-P_{j}\right)$ is the pressure drop across the element from node $i$ to node $j$. Whilst this is clearly an approximation, it forms a useful starting point for the development of more sophisticated models at a later date. At each node, denoted by the index $i$, a number of capillary elements come together and (assuming incompressible flow) mass conservation means that the sum of all flows at each nodal point must add up to zero, i.e.,

$$
\sum_{j=1}^{j=4} Q_{i j}=0
$$

where the $j$ index refers to the four adjacent sites on the 2D lattice. Prescribed pressures are set at sites that are connected to the inflow or outflow faces of the network (in this case at either end of the parent vessel) and these values form part of the boundary conditions for the problem. Application of equation (4) to the whole network of nodes leads to a sparse set of linear pressure equations, the simultaneous solution of which can then be used to calculate elemental flows. In order to apply this model formulation to flow through the vascular networks shown in Fig. 2(a) and (b), the following procedure was adopted:

(i) to be consistent with the theoretical networks generated via the discrete model, the actual flow networks used are obtained by mapping the theoretical networks generated via the discrete model, shown in Fig. 2(a) and (b), onto the 2D lattice template shown in Fig. 3(a). An example mapping is shown in Fig. 4;

(ii) the pressure solution and flow calculations are restricted to the vascular networks themselves, i.e., flow only occurs through the bond elements which are capillaries [as shown in Fig. 3(b)]. Template elements corresponding to nonvascularized tissue [shown dotted in Fig. 3(b)] have no flow through them;

(iii) the uppermost row of bond elements are all assigned the same radius (either 10 or $20 \mu \mathrm{m}$ ) and represent the parent vessel. The global network pressure drop is applied across this capillary vessel only (i.e., in a direction perpendicular to the general direction of vascular growth);

(iv) bond elements corresponding to vascular capillaries are assigned radii from narrow uniform distribution functions [ranges considered were $(2,2.01),(4$, 4.01), and $(6,6.01) \mu \mathrm{m}]$;

(v) the lower boundary is taken to represent the outer surface of the tumour. 
After applying the procedure for a given vascular architecture, and prescribed pressure drop across the parent vessel, the flow field is then calculated by assuming that the capillary network is initially full of pure blood. This gives us the elemental bond flows required for further analysis - in particular, for modelling the flow of chemotherapy drugs through the vascular networks. In the next section, we describe the algorithm developed to facilitate this.

3.2. Chemotherapeutic drug flow modelling. As a first attempt towards modelling the flow of chemotherapy drugs through tumour-induced vascular networks, an algorithm was developed to track concentration profiles of an injected tracer as follows. Having solved for the nodal pressures and elemental flows, a chemotherapy drug at concentration $C_{\max }$ was injected into the upstream end of the parent vessel. At each time step, the following procedure was adopted:

(i) the total amount of drug flowing into each node was calculated;

(ii) perfect mixing was assumed at each node and new drug concentrations were calculated for all outflow bonds based upon nodal values.

Care had to be taken to ensure that mass conservation was satisfied at all timestoo large a time step could result in a mass of drug flowing into a node in excess of that available in the associated capillary element (i.e., too large a time step could lead to cases where $Q_{i j} \Delta t>\pi R_{i j}^{2} L_{i j} c_{i j}$ ). The time step chosen for the simulation was subsequently calculated from:

$$
\Delta t=M I N\left\{\frac{\pi r_{i j}^{2} L_{i j}}{Q_{i j}}\right\}
$$

considered over the whole network of capillaries. Preliminary simulations imposed a no-flow boundary condition at the tumour surface (this corresponds to the lowermost boundary in the figures). In the simulations described later, however, the boundary condition was modified to allow drug uptake by the tumour and we assumed that any drug coming within $40 \mu \mathrm{m}$ of this boundary was immediately removed from the system. The flow simulations were used to compare the drug delivery to the tumour via the two standard treatment regimes of continuous infusion and a one-off, bolus injection.

The most restrictive approximations of the flow model were the following: (i) the blood was considered to be a Newtonian fluid; (ii) the blood vessels were modelled as static, impermeable cylindrical capillaries; and (iii) no reaction kinetics were included in the drug uptake function. Each of these issues has been the subject of recent modelling work at the scale of a single capillary (Armitstead et al., 1996; Dutta and Tarbell, 1996; Pedley and Luo, 1998; Fister and Panetta, 2000) and in future work we will incorporate these effects into our interconnected network flow model. 
Table 1. Base case physiological input data.

\begin{tabular}{lc}
\hline Base case parameter & Value \\
\hline$\mu_{\text {base }}($ Pa.s $)$ & $4 \times 10^{-3}$ \\
$\Delta P_{\text {base }}(\mathrm{Pa})$ & 800 \\
$R_{c, \text { base }}(\mu \mathrm{m})$ & 4 \\
$R_{p v \text {, base }}(\mu \mathrm{m})$ & 10 \\
\hline
\end{tabular}

Table 2. Dimensionless input parameters used for chemotherapy modelling.

\begin{tabular}{cccccc}
\hline & Base run & Run 1 & Run 2 & Run 3 & Run 4 \\
\hline$\mu^{*}$ & 1 & 0.25 & 2 & 1 & 1 \\
$R_{c}^{*}$ & 1 & 1 & 1 & 0.5 & 1.5 \\
\hline
\end{tabular}

Although we cannot directly compare our simulations with experimental results, since to our knowledge no such data exist for drug flow in micro-vascular networks, we do base the parameters for the model on actual physiological data.

3.3. Input data for flow simulations. In total, four suites of flow simulations were undertaken. The first suite focussed upon continuous drug infusion into vasculature 1 (linear TAF source). The second suite corresponded to continuous infusion into vasculature 2 (circular TAF source), whilst for suites 3 and 4, continuous infusion into each vascular network was replaced by a one-off bolus injection. Base case physiological input data corresponding to blood viscosity $\left(\mu_{\text {base }}\right)$, pressure drop across the parent vessel $\left(\Delta P_{\text {base }}\right)$, capillary radii $\left(R_{c \text {,base }}\right)$, and radius of the parent vessel $\left(R_{p v \text {,base }}\right)$ are presented in Table 1. Relevant dimensionless parameters were constructed as follows:

$$
R_{c}^{*}=\frac{R_{c}}{R_{c, \text { base }}} \quad \mu^{*}=\frac{\mu}{\mu_{\text {base }}} \quad t^{*}=\frac{t}{\left(\frac{8 \mu_{\text {base }} L^{2}}{R_{p v, \text { base }}^{2} \Delta P_{\text {base }}}\right)}
$$

where $L$ corresponds to the length of the parent vessel $(2.5 \mathrm{~mm})$ and the denominator in the definition of $t^{*}$ represents the time taken for blood to flow from inlet to outlet along the parent vessel. Sensitivities to mean capillary radius and blood viscosity were considered and the five sets of dimensionless parameter values used are summarized in Table 2. We note that the tabulated parameter values are in keeping with experimental data and physiological observations [see, for example, Levick (1998)].

Throughout each simulation, data was collected corresponding to the total mass of drug in the vascular network $\left(M^{*}\right)$, the drug concentration in blood emerging from the downstream end of the parent vessel $\left(C^{*}\right)$ and the total mass taken up by 
the tumour $\left(M T^{*}\right)$. These dimensionless variables are defined as follows:

$$
\begin{aligned}
M^{*} & =\frac{\text { Total drug mass in capillaries }}{\text { Drug mass in parent vessel when } c=C_{\max }} \\
M T^{*} & =\frac{\text { Total drug mass taken up by tumour }}{\text { Drug mass in parent vessel when } c=C_{\max }} \\
C^{*} & =\frac{\text { Drug concentration in outlet bond }}{C_{\max }}
\end{aligned}
$$

\subsection{Simulation results.}

3.4.1. Continuous infusion into vasculature 1 (linear TAF source). The first set of results corresponds to continuous drug infusion into vascular network 1 shown in Fig. 2, which was generated by a linear source of TAF. A chemotherapy drug of concentration $C_{\max }$ is fed into the parent vessel at $t^{*}=0$. Sequential drug concentration profiles for the base case simulation are shown in Fig. 5. In this, and all subsequent images, the five colours represent different drug concentrations $c(x, y, t)$ as follows:

$$
\begin{gathered}
\text { Dark blue }-0.0 \leq c(x, y, t) / C_{\max }<0.001 ; \\
\text { Light blue }-0.001 \leq c(x, y, t) / C_{\max }<0.1 ; \\
\text { White }-0.1 \leq c(x, y, t) / C_{\max }<0.5 ; \\
\text { Pink }-0.5 \leq c(x, y, t) / C_{\max }<0.9 ; \\
\text { Red }-0.9 \leq c(x, y, t) / C_{\max } \leq 1.0,
\end{gathered}
$$

where $C_{\max }$ corresponds to the maximum (input) drug concentration. Note that the entire vasculature was assumed to have been flowing 'clean' blood at $t^{*}<0$, after which the drug was introduced into the left-hand side of the parent vessel.

Figure 5 shows the simulation results for continuous infusion into vasculature 1 enabling us to monitor the flow of drug from the parent vessel to the tumour. The magnitude of the flow into successive downstream capillary branches decreases as the upstream supply diminishes. This effect is evident in branches 1 and 2, which supply drug to the tumour [Fig. 5(a)-(c)]. Moving further downstream, however, the vascular network is such that blood flow in the capillaries begins to flow from the tumour towards the parent vessel. Hence, instead of supplying drug to the tumour, branches 3-5 actually remove drug from the capillary network [Fig. 5(d)(i)], thereby reducing its efficacy. Indeed, for this set of input data, $420 \mathrm{~s}\left(t^{*}=168\right)$ of continuous infusion is required for a drug concentration of $0.1 \% C_{\max }$ to reach the tumour. 


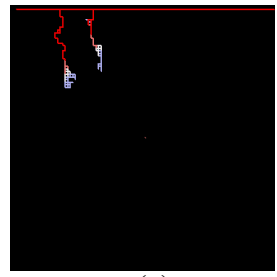

(a)

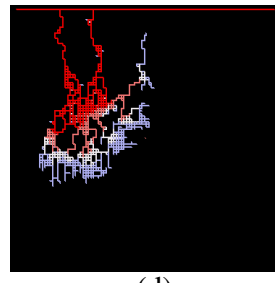

(d)

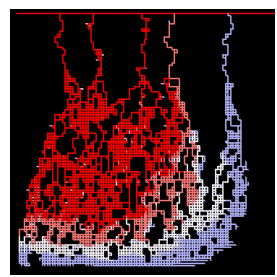

(g)

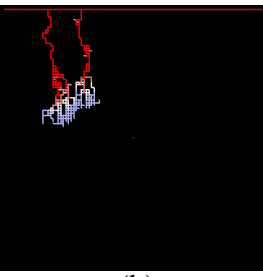

(b)

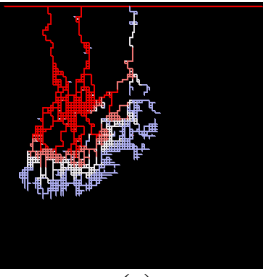

(e)

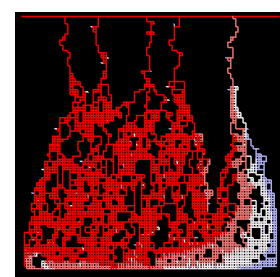

(h)

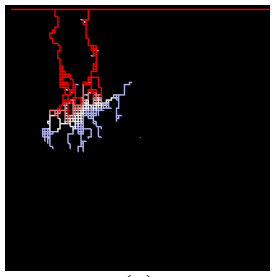

(c)

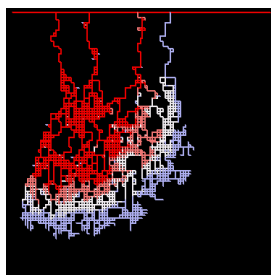

(f)

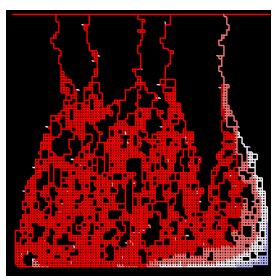

(i)

Figure 5. Snapshots in time of the drug concentration as it flows from the parent vessel into the vascular network (vasculature 1) under continuous infusion for times (a) $t^{*}=2.4$; (b) $t^{*}=7.2$; (c) $t^{*}=12$; (d) $t^{*}=24$; (e) $t^{*}=36$; (f) $t^{*}=72$; (g) $t^{*}=168$; (h) $t^{*}=360$; (i) $t^{*}=624$. The various colours correspond to different drug concentrations (see text for details). This represents the base case simulation with mean capillary radius $R_{c}^{*}=1$ and viscosity $\mu^{*}=1$.

We now examine the effect of blood viscosity upon drug delivery and uptake by the tumour. The simulation results shown in Fig. 6 demonstrate that the drug reaches the tumour sooner when the blood viscosity is decreased. This may be inferred from the inverse relationship between elemental flow and fluid viscosity, coupled with the linear nature of the pressure equations. A similar effect is observed by increasing the global pressure drop across the parent vessel (results not shown). The qualitative form of the profiles of drug mass and tumour uptake shown in Fig. 6 are typical of all cases. The total drug mass increases initially as the vasculature fills, and reaches a steady state as the vasculature becomes saturated with drug. Conversely, the drug delivered to the tumour is small initially, and begins to increase at a constant rate as the vasculature fills.

We next examine the effect of changing the mean capillary radius of vasculature 1 and how this affects drug delivery. Figure 7 shows the results of drug delivery into capillaries with a mean radius of $6 \mu \mathrm{m}\left(R_{c}^{*}=1.5\right), 4 \mu \mathrm{m}\left(R_{c}^{*}=1\right)$ and $2 \mu \mathrm{m}$ $\left(R_{c}^{*}=0.5\right)$. As the radius decreases the flow rate and, consequently, drug uptake by the tumour decreases. Hence for narrower capillaries infusion would have to 


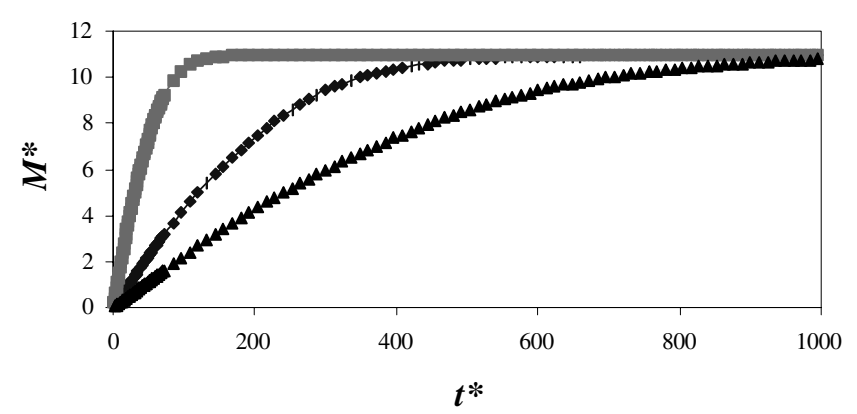

(a)

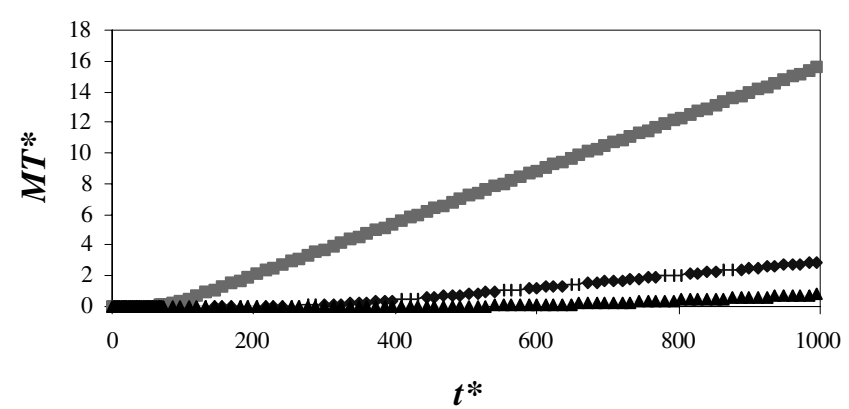

(b)

Figure 6. Plots of the total drug mass in the vasculature and the mass uptake by the tumour for three different values of viscosity under continuous infusion of drug into vasculature 1 with $R_{c}^{*}=1$. (a) Total drug mass $\left(M^{*}\right)$ in vasculature; (b) mass uptake $\left(M T^{*}\right)$ by tumour. $\mu^{*}=2\left(\boldsymbol{\Lambda}\right.$, lower curve), $\mu^{*}=1(\diamond$, middle curve $), \mu^{*}=0.25$ ( $\boldsymbol{\square}$, upper curve).

continue for many hours/days before significant tumour uptake could occur. However, as Fig. 7 shows, this effect is nonlinear due to the relationships between bond radius, bond volume, bond conductivity and the connectedness of the network. This means that the effect of variations in capillary radii cannot simply be inferred from the base case simulation (as could be done for blood viscosity and parent vessel pressure drop earlier).

We have also considered the effect of varying the radius of the parent vessel. Increasing the radius to $20 \mu \mathrm{m}$, twice that used in the base case, gives results that are similar to the base case. The fact that the pressure drop across the parent vessel was the same in both cases ( $800 \mathrm{~Pa})$, coupled with the fact that the ratio of capillary flow to parent vessel flow scales as $\left\langle r_{\text {cap }}\right\rangle^{4} / R_{\mathrm{pv}}^{4}$, means that the pressure solution is largely unaffected by varying the radius of the parent vessel within reasonable limits. Hence, the actual volume of blood flowing through the parent vessel per unit time would appear to be less important than the associated pressure drop across it.

3.4.2. Continuous infusion into vasculature 2 (circular TAF source). Figure 8 shows the flow profile for continuous drug infusion into vasculature 2 [shown in Fig. 2(b)], which is generated by a circular source of TAF. Again, the upstream 


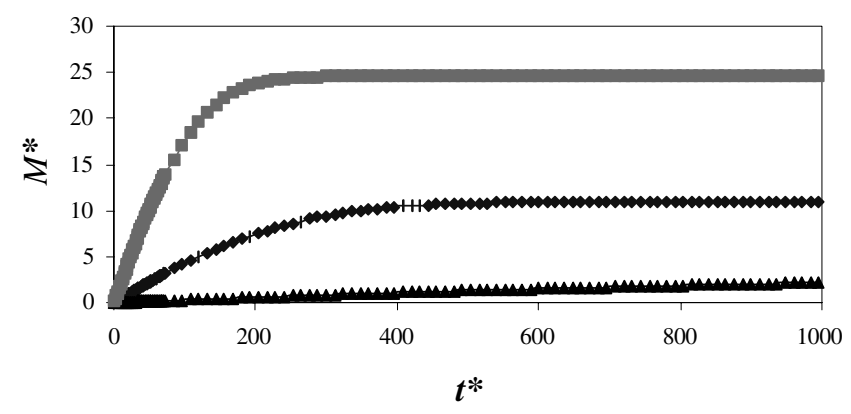

(a)

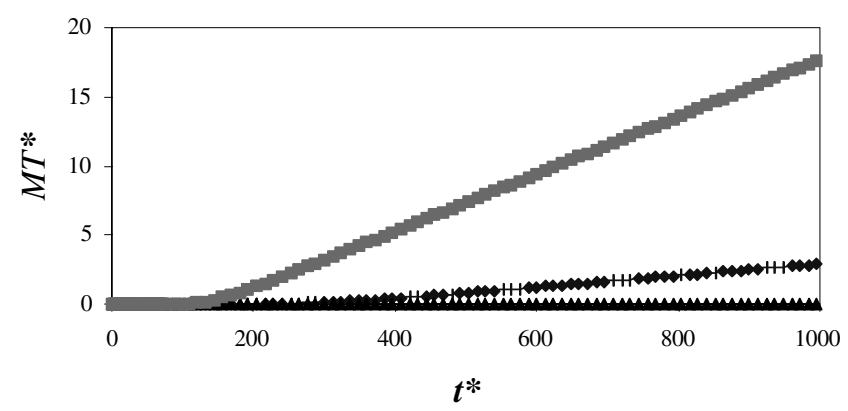

(b)

Figure 7. Plots of the total drug mass in the vasculature and the mass uptake by the tumour for three different values of mean capillary radius under continuous infusion of drug into vasculature 1 with $\mu^{*}=1$. (a) Total drug mass $\left(M^{*}\right)$ in vasculature; (b) mass uptake $\left(M T^{*}\right)$ by tumour. $R_{c}^{*}=0.5(\boldsymbol{\Lambda}$, lower curve $), R_{c}^{*}=1(\diamond$, middle curve $), R_{c}^{*}=1.5(\boldsymbol{\square}$, upper curve).

branches on the parent blood vessel have blood flowing towards the tumour, while flow is away from the tumour in branches further downstream. In comparison to vasculature 1 , considered earlier, this network has a much higher degree of connectedness (anastamosis density), and in particular, capillary branches form loops much nearer to the parent vessel. These effects arise because the circular geometry tends to focus the growing capillaries, and has significant implications for the resulting blood flow. The large number of interconnections significantly reduce the bulk flow, with a consequent reduction in drug delivery to the tumour. This is quantified in Fig. 9, where drug delivery to the tumour via the two vascular networks is compared: the two cases differ by several orders of magnitude. Sensitivities to blood viscosity (Fig. 10) and mean capillary radius (Fig. 11) are similar to those described earlier. Comparison of Figs 6 and 10, or Figs 7 and 11, is instructive: compared to vasculature 1 , the capillaries in vasculature 2 contain almost as much drug. However the amount of the drug actually taken up by the tumour is much larger in vasculature 1 . This is because almost all of the drug in vasculature 2 is in capillaries far from the tumour, with very little blood flow near the tumour itself. These results emphasize the importance of the capillary network structure. 


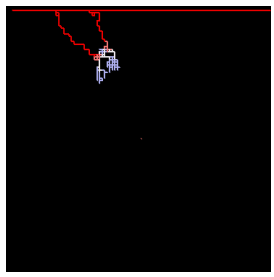

(a)

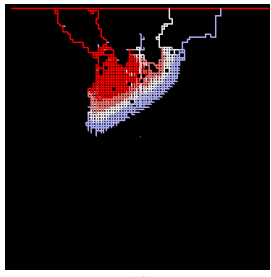

(d)

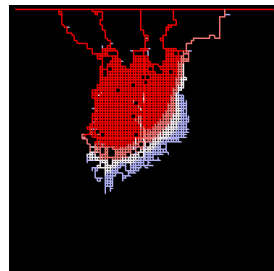

(g)

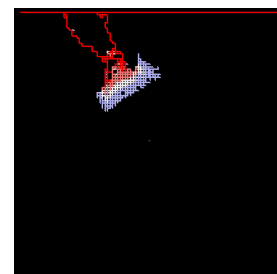

(b)

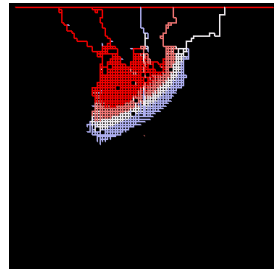

(e)

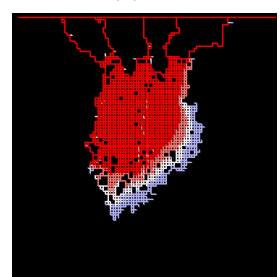

(h)

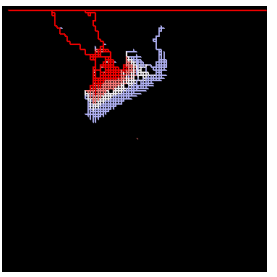

(c)

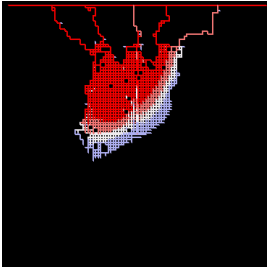

(f)

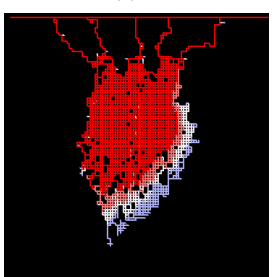

(i)

Figure 8. Snapshots in time of the drug concentration as it flows from the parent vessel into the vascular network (vasculature 2) under continuous infusion for times (a) $t^{*}=2.4$; (b) $t^{*}=7.2$; (c) $t^{*}=12$; (d) $t^{*}=24$; (e) $t^{*}=36$; (f) $t^{*}=72$; (g) $t^{*}=168$; (h) $t^{*}=360$; (i) $t^{*}=624$. The various colours correspond to different drug concentrations (see text for details). This represents the base case simulation with mean capillary radius $R_{c}^{*}=1$ and viscosity $\mu^{*}=1$ (cf. Fig. 5).

3.4.3. Bolus injection into vasculature 1 (linear TAF source). In contrast to the continuous infusion results discussed earlier, the third suite of simulations followed the dispersion of a bolus injection of chemotherapy drug into vasculature 1 , delivered at $t^{*}=0$ and lasting $30 \mathrm{~s}\left(t^{*}=12\right)$. Figure 12 shows the corresponding concentration profiles.

Whilst the overall structure of the advancing drug fronts correspond precisely to those depicted in the continuous infusion runs, there is now an additional effect that merits closer examination. Post-injection, the re-invasion of pure blood into the vascular network leads to dilution of the drug. This may have serious consequences for chemotherapy strategies-for example, if successful treatment depends upon a threshold drug concentration reaching the tumour, then this may not be possible under certain circumstances.

One additional variable may also play an important role in determining the efficacy of a chemotherapy treatment, namely the duration of the bolus injection itself. In order to investigate this issue, a base-case simulation was repeated using an injection time of only $3 \mathrm{~s}\left(t^{*}=1.2\right)$. The reduced injection period meant that 


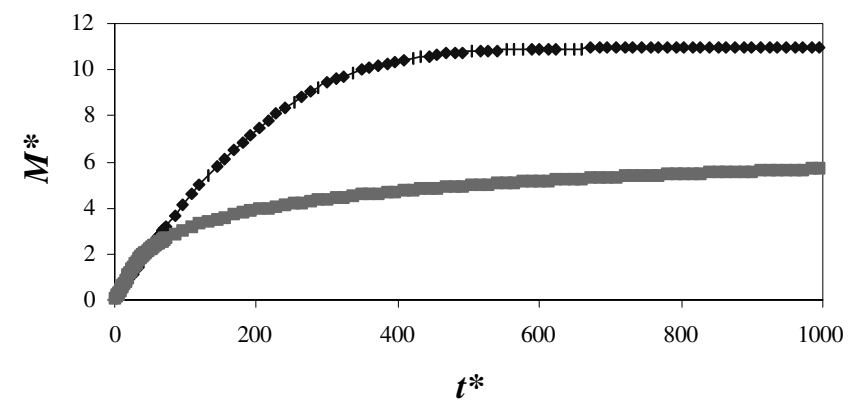

(a)

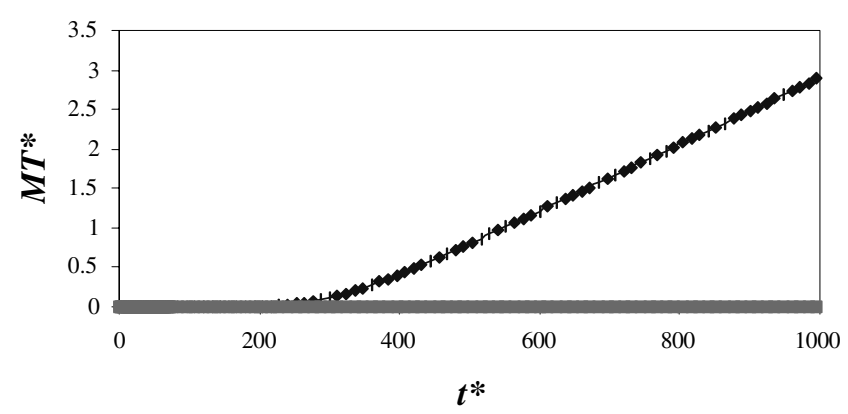

(b)

Figure 9. Comparison of drug delivery in vasculature 1 with drug delivery in vasculature 2 under continuous infusion with $R_{c}^{*}=1$ and $\mu^{*}=1$ (base case). (a) Total drug mass $\left(M^{*}\right)$ in both vasculatures; (b) mass uptake $\left(M T^{*}\right)$ by tumour in both vasculatures. Vasculature 1 $(\downarrow$, upper curve), vasculature $2(\boldsymbol{\square}$, lower curve).

dilution had an even more dramatic effect, with very little of the drug reaching the tumour (not illustrated).

3.4.4. Bolus injection into vasculature 2 (circular TAF source). For vasculature 2, the results for bolus injection of drug are even more striking (Fig. 13). The large number of interconnections between capillaries-even quite far from the tumour-means that there is very little flow in the lower part of the network, and the bolus of drug essentially bypasses the tumour. This is emphasized by comparison of drug delivery via the two vasculatures for the bolus injection case, in Fig. 14.

\section{DISCUSSION}

Mathematical modelling of tumour-induced angiogenesis has been a very active research area in recent years. This has lead to a detailed understanding of the way in which the migration of endothelial cells is guided and governed by gradients of angiogenic chemicals (TAFs) and extracellular matrix components and how these combine to generate the vascular networks seen around solid tumours. Building on 


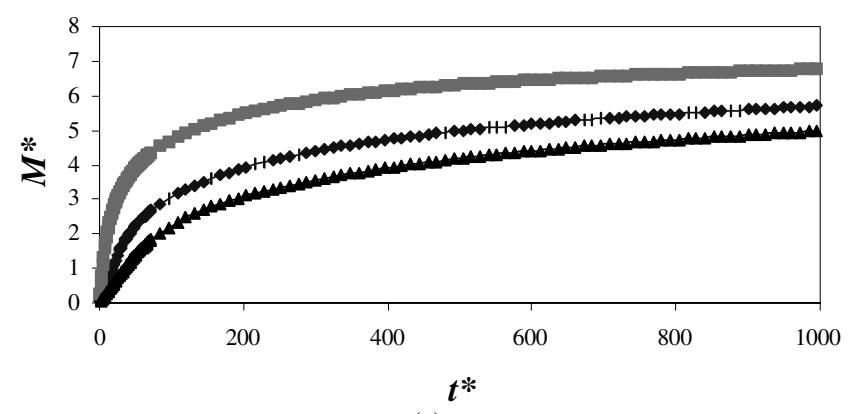

(a)

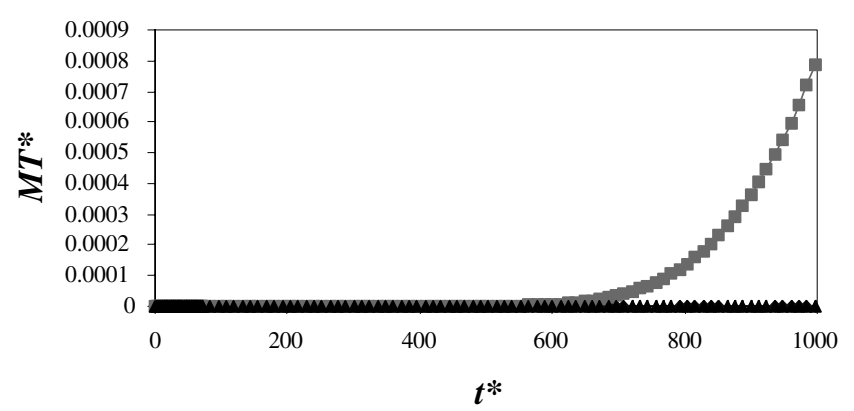

(b)

Figure 10. Plots of the total drug mass in the vasculature and the mass uptake by the tumour for three different values of viscosity under continuous infusion of drug into vasculature 2 with $R_{c}^{*}=1$. (a) Total drug mass $\left(M^{*}\right)$ in vasculature; (b) mass uptake $\left(M T^{*}\right)$ by tumour. $\mu^{*}=2(\boldsymbol{\Lambda}$, lower curve $), \mu^{*}=1(\boldsymbol{\diamond}$, middle curve $), \mu^{*}=0.25(\boldsymbol{\square}$, upper curve $)$.

this previous work, we have considered, for the first time, flow through the vascular network and subsequently the way in which the structure of the vascular network affects the flow within it. This has implications for the delivery of chemotherapeutic drugs to the tumour. In order to achieve this we have adapted computational techniques normally used to simulate the flow of oil and water through interconnected networks of pore spaces found in rock structures. Using this approach, we have been able to simulate flow through vascular networks generated from a mathematical model (Anderson and Chaplain, 1998). In particular, we considered two network structures differing in connectedness and examined how the networks alter the flow of chemotherapeutic drug delivery to the tumour. In order to examine the effects of different drug delivery regimes on drug uptake by the tumour, we simulated a continuous infusion regime and a bolus injection regime. We also considered the effects of varying key flow parameters and capillary radii on drug uptake.

The two network structures we considered are shown in Fig. 2(a) and (b) and represent capillary networks which have grown in response to two tumours of different size, one larger than the other i.e., vasculature 1 [large linear tumour, Fig. 2(a)] and vasculature 2 [small circular tumour, Fig. 2(b)]. 


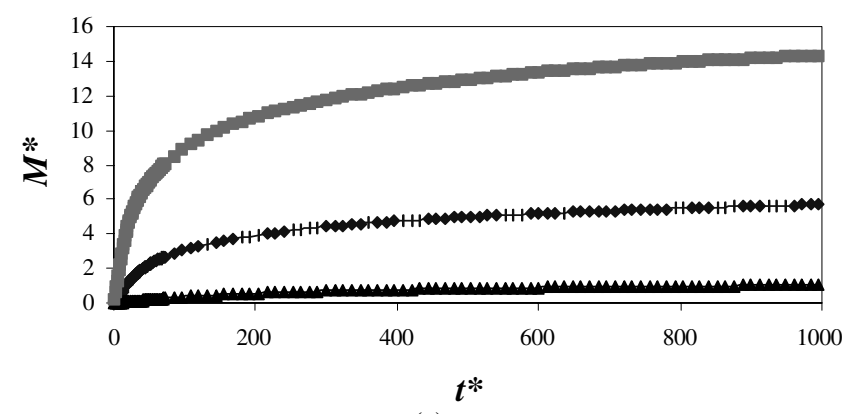

(a)

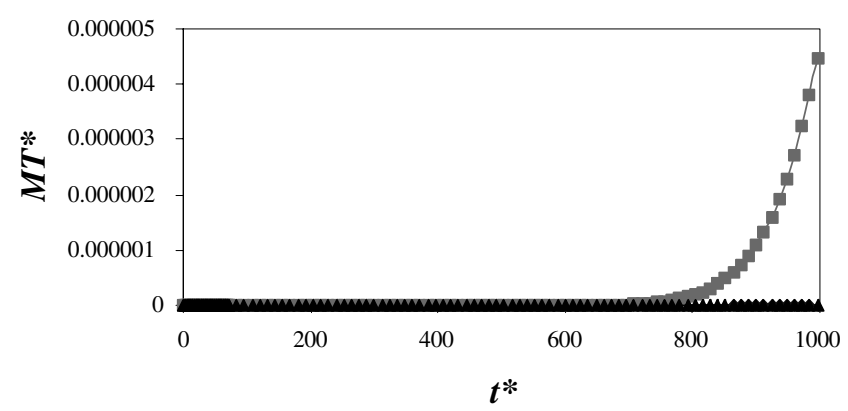

(b)

Figure 11. Plots of the total drug mass in the vasculature and the mass uptake by the tumour for three different values of mean capillary radius under continuous infusion of drug into vasculature 2 with $\mu^{*}=1$. (a) Total drug mass $\left(M^{*}\right)$ in vasculature; (b) mass uptake (MT*) by tumour. $R_{c}^{*}=0.5$ ( $\mathbf{\Lambda}$, lower curve), $R_{c}^{*}=1\left(\diamond\right.$, middle curve), $R_{c}^{*}=1.5$ ( $\boldsymbol{\square}$, upper curve).

For both vasculatures we examined the effects of varying blood viscosity, pressure drop across the parent vessel and mean capillary radii (both in the parent vessel and in the vasculature itself). The relationship between these parameters is given by equation (3) governing the flow rate $\left(Q_{i j}\right)$ in the $i j$ th capillary element (joining nodes $i$ and $j$ ): as is expected from this equation, decreasing the blood viscosity $(\mu)$ led to an increase in flow rate and consequently an increase in drug uptake by the tumour (see Figs 6 and 10 for results). Similar results were obtained by varying the pressure drop across the parent vessel $(\Delta P)$, i.e., increasing the pressure drop led to an increase in drug uptake by the tumour (results not shown).

Increasing the capillary radii of the two vasculatures also produced an increase in the flow rate and consequently an increase in drug uptake by the tumour. However, as Figs 7 and 11 show, this effect is nonlinear due to the relationships between bond radius, bond volume, bond conductivity and the connectedness of the network. Variation in the radius of the parent vessel had little effect on the results.

For both vasculatures we examined two different drug delivery regimes, either a continuous infusion or a bolus injection. The results showed that there are two important factors in determining how much drug reaches the tumour: (i) the vascular structure and (ii) the particular mode of delivery. These results were quantified 


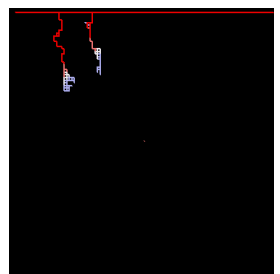

(a)

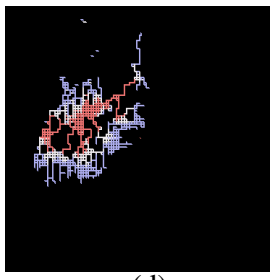

(d)

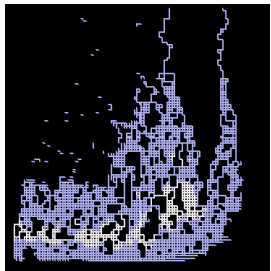

(g)

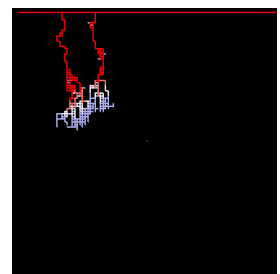

(b)

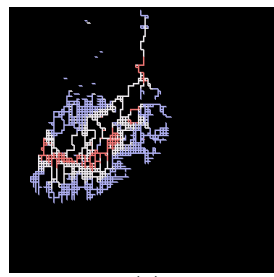

(e)

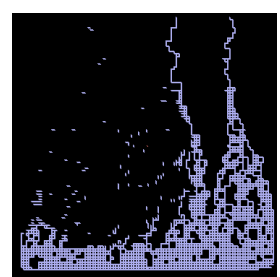

(h)

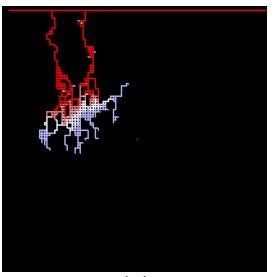

(c)

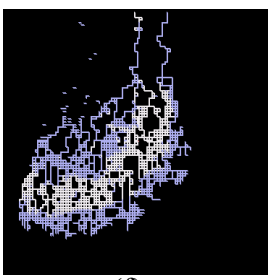

(f)

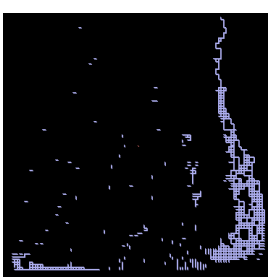

(i)

Figure 12. Snapshots in time of the drug concentration as it flows from the parent vessel into the vascular network (vasculature 1) under a bolus injection for times (a) $t^{*}=2.4$; (b) $t^{*}=7.2$; (c) $t^{*}=12$; (d) $t^{*}=24$; (e) $t^{*}=36$; (f) $t^{*}=72$; (g) $t^{*}=168$; (h) $t^{*}=360$; (i) $t^{*}=624$. The various colours correspond to different drug concentrations (see text for details). This represents the base case simulation with mean capillary radius $R_{c}^{*}=1$ and viscosity $\mu^{*}=1$.

and summarized in Figs 9 and 14 where we see that, although the total mass of drug within each vasculature is similar under all delivery regimes, vasculature 1 permits a far greater drug uptake by the tumour. This is because almost all of the drug in vasculature 2 is in capillaries far from the tumour, with very little blood flow near the tumour to deliver the drug.

If we consider drug delivery to the vascular network via bolus injection, there is an additional effect that merits closer examination. Post-injection, the re-invasion of pure blood into the vascular network leads to dilution of the drug. We also found that the duration of the bolus injection affects the level of drug dilution This has serious implications for chemotherapy strategies. For example, if successful treatment depends upon a threshold drug concentration reaching the tumour, then this may not be possible under certain circumstances.

The key result of our work is that the highly interconnected vascular structures around a tumour cause relatively low rates of drug delivery to the tumour itself, with the vast majority of drug simply by-passing the tumour and returning to the parent vessel. This is most pronounced for the dense capillary network generated 


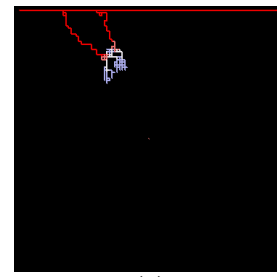

(a)

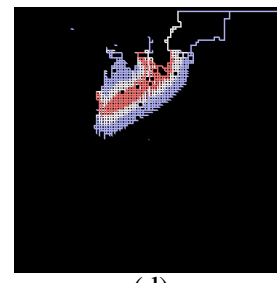

(d)

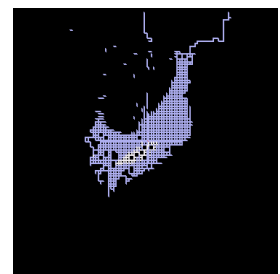

(g)

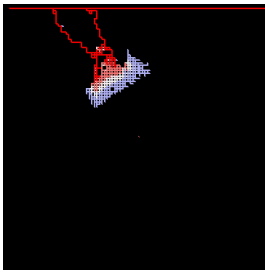

(b)

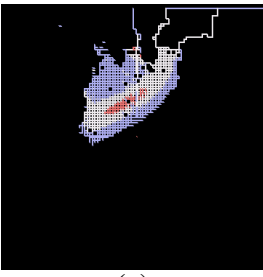

(e)

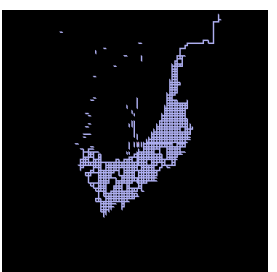

(h)

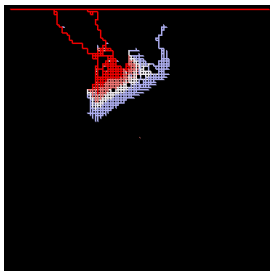

(c)

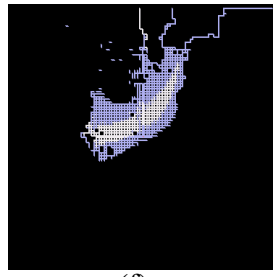

(f)

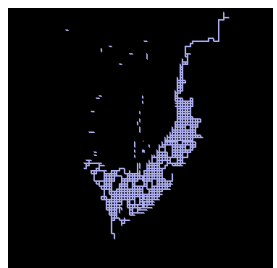

(i)

Figure 13. Snapshots in time of the drug concentration as it flows from the parent vessel into the vascular network (vasculature 2) under a bolus injection for times (a) $t^{*}=2.4$; (b) $t^{*}=7.2$; (c) $t^{*}=12$; (d) $t^{*}=24$; (e) $t^{*}=36$; (f) $t^{*}=72$; (g) $t^{*}=168$; (h) $t^{*}=360$; (i) $t^{*}=624$. The various colours correspond to different drug concentrations (see text for details). This represents the base case simulation with mean capillary radius $R_{c}^{*}=1$ and viscosity $\mu^{*}=1$.

by a small circular tumour implant (vasculature 2). Here, our simulation of a bolus injection of drug shows that it entirely by-passes the tumour. These results have important implications for chemotherapy strategies, suggesting that the structure of the vasculature around the tumour should be considered when planning chemotherapy, which is not current practice. Clearly our model has the potential to predict patient specific chemotherapeutic strategies i.e., appropriate drug dosage and delivery time.

The model could also be used to study the actual nutrient supply (e.g., of bloodborne oxygen) to the tumour via the vascular network and could be readily adapted to investigate flow from the tumour to the main blood vessel (e.g., of waste products, certain soluble growth factors and tumour cells themselves). Additional natural extensions to model include: (i) correlating blood viscosity with haematocrit (the percentage of blood volume comprising red blood cells), capillary radius, and shear rate; (ii) modelling blood capillaries as collapsible, permeable tubes instead of rigid, impermeable cylinders; and (iii) including reaction kinetics into the drug uptake function. 


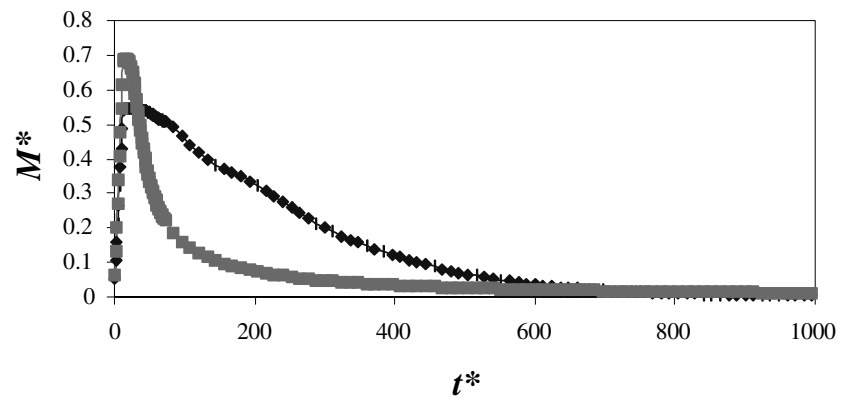

(a)

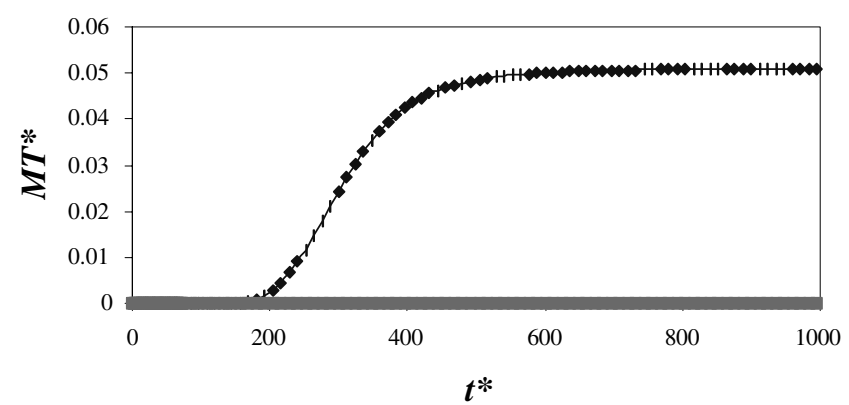

(b)

Figure 14. Comparison of drug delivery in vasculature 1 with drug delivery in vasculature 2 under a bolus injection with $R_{c}^{*}=1$ and $\mu^{*}=1$ (base case). (a) Total drug mass $\left(M^{*}\right)$ in both vasculatures; (b) mass uptake $\left(M T^{*}\right)$ by tumour in both vasculatures. Vasculature 1 ( $\downarrow$, upper curve), vasculature $2(\boldsymbol{\square}$, lower curve).

Of course, the most immediate extension of the model will be to model flow processes in 3D vasculatures. Preliminary results of Chaplain and Anderson (1999) in generating $3 \mathrm{D}$ vasculatures in a $2 \mathrm{~mm}$ cube of tissue suggest that the number of anastomoses (loops) formed is reduced when compared with equivalent 2D simulation results. The loops also form closer to the tumour. If we extrapolate the $2 \mathrm{D}$ flow results of this paper, this would indicate that flow to the tumour in a 3D network might actually increase. However, given the inherent nonlinearities involved, until more extensive modelling and flow simulations are carried out in 3D, this remains an interesting conjecture.

\section{ACKNOWLEDGEMENTS}

The work of AA was partly supported by BBSRC Grant MMI09008 and by a Personal Research Fellowship from the RSE. This work was supported in part by SHEFC research development grants 107 (Centre for Theoretical Modelling in Medicine) and 099 (SIMBIOS Centre). JAS thanks Oliver Jensen for helpful advice. 


\section{REFERENCES}

Alberts, B., D. Bray, J. Lewis, M. Raff, K. Roberts and J. D. Watson (1994). Molecular Biology of the Cell, 3rd edn, New York: Garland Publishing.

Anderson, A. R. A. and M. A. J. Chaplain (1998). Continuous and discrete mathematical models of tumour-induced angiogenesis. Bull. Math. Biol. 60, 857-899.

Anderson, A. R. A., B. D. S. Sleeman, I. M. Young and B. S. Griffiths (1997). Nematode movement along a chemical gradient in a structurally heterogeneous environment: II. Theory. Fundam. Appl. Nematol. 20, 165-172.

Anderson, A. R. A., M. A. J. Chaplain, A. L. Newman, R. J. C. Steele and A. M. Thompson (2000). Mathematical modelling of tumour invasion and metastasis. J. Theor. Med. 2, 129-154.

Andreasen, P. A., L. Kjøller, L. Christensen and M. J. Duffy (1997). The urokinase-type plasminogen activator system in cancer metastasis: a review. Int. J. Cancer 72, 1-22.

Armitstead, J. P., C. D. Bertram and O. E. Jensen (1996). A study of the bifurcation behaviour of a model of flow through a collapsible tube. Bull. Math. Biol. 58, 611-641.

Arnold, F. and D. C. West (1991). Angiogenesis in wound healing. Pharmacol. Ther. 52, 407-422.

Ausprunk, D. H. and J. Folkman (1977). Migration and proliferation of endothelial cells in preformed and newly formed blood vessels during tumour angiogenesis. Microvasc. Res. 14, 53-65.

Baish, J. W., Y. Gazit, D. A. Berk, M. Nozue, L. T. Baxter and R. K. Jain (1996). Role of tumour vascular architecture in nutrient and drug delivery: an invasion percolationbased network model. Microvasc. Res. 51, 327-346.

Baish, J. W., P. Netti and R. K. Jain (1997). Transmural coupling of fluid flow in microcirculatory network and interstitium in tumours. Microvasc. Res. 53, 128-141.

Balding, D. and D. L. S. McElwain (1985). A mathematical model of tumour-induced capillary growth. J. Theor. Biol. 114, 53-73.

Bikfalvi, A. (1995). Significance of angiogenesis in tumour progression and metastasis. Euro. J. Cancer 31A, 1101-1104.

Bowersox, J. C. and N. Sorgente (1982). Chemotaxis of aortic endothelial cells in response to fibronectin. Cancer Res. 42, 2547-2551.

Chaplain, M. A. J. and B. D. Sleeman (1990). A mathematical model for the production and secretion of tumour angiogenesis factor in tumours. IMA J. Math. Appl. Med. Biol. 7, 93-108.

Chaplain, M. A. J. and A. M. Stuart (1993). A model mechanism for the chemotactic response of endothelial cells to tumour angiogenesis factor. IMA J. Math. Appl. Med. Biol. 10, 149-168.

Chaplain, M. A. J. (1996). Avascular growth, angiogenesis and vascular growth in solid tumours: the mathematical modelling of the stages of tumour development. Math. Comp. Modell. 23, 47-87.

Chaplain, M. A. J. and A. R. A. Anderson (1999). Modelling the growth and form of capillary networks, in On Growth and Form: Spatio-temporal Pattern Formation in Biology, M. A. J. Chaplain, G. D. Singh and J. C. McLachlan (Eds), Chichester: Wiley, pp. 225-250. 
Cliff, W. J. (1963). Observations on healing tissue: A combined light and electron microscopic investigation. Trans. Roy. Soc. Lond. B246, 305-325.

Dodd, C. G. and O. G. Kiel (1959). Evaluation of Monte Carlo methods in studying fluidfluid displacement and wettability in porous rock. J. Phys. Chem. 63, 1646.

Dullien, F. A. L. (1992). Porous Media, Fluid Transport and Pore Structure, 2nd edn, New York: Academic Press Inc.

Dutta, A. and J. M. Tarbell (1996). Influence of non-Newtonian behaviour of blood on flow in an elastic artery model. J. Biomech. Eng.-Trans. ASME 118, 111-119.

Ellis, L. E. and I. J. Fidler (1995). Angiogenesis and breast cancer metastasis. Lancet 346, 388-389.

Fatt, I. (1956). The network model of porous media. Trans. AIME 207, 144.

Fister, K. R. and J. C. Panetta (2000). Optimal control applied to cell-cycle-specific cancer chemotherapy. Siam J. Appl. Math. 60, 1059-1072.

Folkman, J. (1985). Tumor angiogenesis. Adv. Cancer Res. 43, 175-203.

Folkman, J. and M. Klagsbrun (1987). Angiogenic factors. Science 235, 442-447.

Folkman, J. and H. Brem (1992). Angiogenesis and inflammation, in Inflammation: Basic Principles and Clinical Correlates, 2nd edn, J. I. Gallin, I. M. Goldstein and R. Snyderman (Eds), New York: Raven Press.

Folkman, J. (1995). Angiogenesis in cancer, vascular, rheumatoid and other disease. Nat. Med. 1, 21-31.

Gasparini, G. (1995). Tumour angiogenesis as a prognostic assay for invasive ductal breastcarcinoma. J. Natl. Cancer Inst. 87, 1799-1801.

Gasparini, G. and A. L. Harris (1995). Clinical importance of the determination of tumour angiogenesis in breast-cancer-much more than a new prognostic tool. J. Clin. Oncol. 13, 765-782.

Gödde, R. and H. Kurz (2001). Structural and biophysical simulation of angiogenesis and vascular remodelling. Dev. Dyn. 220, 387-401.

Graham, C. H. and P. K. Lala (1992). Mechanisms of placental invasion of the uterus and their control. Biochem. Cell Biol. 70, 867-874.

Harris, A. L., S. Fox, R. Bicknell, R. Leek and K. Gatter (1994). Tumour angiogenesis in breast-cancer-prognostic factor and therapeutic target. J. Cellular Biochem. S18D SID, 225.

Harris, A. L., H. T. Zhang, A. Moghaddam, S. Fox, P. Scott, A. Pattison, K. Gatter, I. Stratford and R. Bicknell (1996). Breast cancer angiogenesis—new approaches to therapy via anti-angiogenesis, hypoxic activated drugs, and vascular targeting. Breast Cancer Res. Treat. 38, 97-108.

Harris, A. L. (1997). Antiangiogenesis for cancer therapy. Lancet 349 (suppl. II), 13-15.

Herblin, W. F. and J. L. Gross (1994). Inhibition of angiogenesis as a strategy for tumourgrowth control. Mol. Chem. Neuropathol. 21, 329-336.

Holmes, M. J. and B. D. Sleeman (2000). A mathematical model of tumour angiogenesis incorporating cellular traction and viscoelastic effects. J. Theor. Biol. 202, 95-112.

Honda, H. and K. Yoshizato (1997). Formation of the branching pattern of blood vessels in the wall of the avian yolk sac studied by a computer simulation. Dev. Growth Differ. 39, 581-589. 
Itoh, J., K. Yasumura, T. Takeshita, H. Ishikawa, H. Kobayashi, K. Ogawa, K. Kawai, A. Serizana and R. Y. Osamura (2000). Three-dimensional imaging of tumor angiogenesis. Anal. Quant. Cytol. Histol. 22, 85-90.

Levick, J. R. (1998). An Introduction to Cardiovascular Physiology, Oxford: ButterworthHeinemann.

Levin, M., B. Dawant and A. S. Popel (1986). Effect of dispersion on vessel diameters and lengths in stochastic networks. I. Modelling of microvascular haematocrit distribution. Microvasc. Res. 31, 223-234.

Levine, H. A., S. Pamuk, B. D. Sleeman and M. Nilsen-Hamilton (2001). Mathematical modelling of capillary formation and development in tumor angiogenesis: penetration into the stroma. Bull. Math. Biol. 63, 801-863.

Liotta, L. A., C. N. Rao and S. H. Barsky (1983). Tumour invasion and the extracellular matrix. Lab. Invest. 49, 636-649.

Madri, J. A. and B. M. Pratt (1986). Endothelial cell-matrix interactions: in vitro models of angiogenesis. J. Histochem. Cytochem. 34, 85-91.

Matrisian, L. M. (1992). The matrix-degrading metalloproteinases. Bioessays 14, 455-463.

Mitchell, A. R. and D. F. Griffiths (1980). The Finite Difference Method in Partial Differential Equations, Chichester: Wiley.

McDougall, S. R. and K. S. Sorbie (1997). The application of network modelling techniques to multiphase flow in porous media. Petroleum Geosci. 3, 161-169.

Muthukkaruppan, V. R., L. Kubai and R. Auerbach (1982). Tumor-induced neovascularization in the mouse eye. J. Natl. Cancer Inst. 69, 699-705.

Nekka, F., S. Kyriacos, C. Kerrigan and L. Cartilier (1996). A model of growing vascular structures. Bull. Math. Biol. 58, 409-424.

Norton, J. A. (1995). Tumor angiogenesis: the future is now. Ann. Surg. 222, 693-694.

Orme, M. E. and M. A. J. Chaplain (1996). A mathematical model of the first steps of tumour-related angiogenesis: capillary sprout formation and secondary branching. IMA J. Math. App. Med. Biol. 13, 73-98.

Paweletz, N. and M. Knierim (1989). Tumor-related angiogenesis. Crit. Rev. Oncol. Hematol. 9, 197-242.

Pedley, T. J. and X. Y. Luo (1998). Modelling flow and oscillations in collapsible tubes. Theor. Comput. Fluid Dyn. 10, 277-294.

Pepper, M. S. (2001). Role of the matrix metalloproteinase and plasminogen activatorplasmin systems in angiogenesis. Arterioscler. Thromb. Vasc. Biol. 21, 1104-1117.

Pettet, G., M. A. J. Chaplain, D. L. S. McElwain and H. M. Byrne (1996). On the role of angiogenesis in wound healing. Proc. Roy. Soc. Lond. B 263, 1487-1493.

Perumpanani, A. J., J. A. Sherratt, J. Norbury and H. M. Byrne (1996). Biological inferences from a mathematical model of malignant invasion. Invasion Metastasis 16, 209-221.

Price, R. J. and T. C. Skalak (1995). A circumferential stress-growth rule predicts arcade arteriole formation in a network model. Microcirculation 2, 41-51.

Pries, A. R., T. W. Secomb and P. Gaehtgens (1998). Structural adaptation and stability of microvascular networks: theory and simulations. Am. J. Physiol. 275, H349-H360. 
Pries, A. R., T. W. Secomb, P. Gaehtgens and J. F. Gross (1990). Blood flow in microvascular networks: experiments and simulations. Circ. Res. 67, 826-834.

Rose, W. (1957). Studies of waterflood performance, in Use of Network Models Illinois State Geology Survey, Vol. 3, Illinois: Circ. No. 237, Urbana.

Schoefl, G. I. (1963). Studies on inflammation III. Growing capillaries: Their structure and permeability. Virchows Arch. Pathol. Anat. 337, 97-141.

Schor, A. M., S. L. Schor and R. Baillie (1999). Angiogenesis experimental data relevant to theoretical analysis, in On Growth and Form: Spatio-temporal Pattern Formation in Biology, M. A. J. Chaplain, G. D. Singh and J. C. McLachlan (Eds), Chichester: Wiley, pp. 202-224.

Schor, A. M., S. L. Schor, A. R. A. Anderson and M. A. J. Chaplain (2002). Chemokinesis and chemotaxis within a three-dimensional collagen matrix: context modulation of fibroblast motogenic response to wound healing cytokines. J. Cell. Biol. (submitted)

Schmid-Schonbein, G. W., R. Skalak, S. Usami and S. Chien (1980). Cell distribution in capillary networks. Microvasc. Res. 19, 18-44.

Schmid-Schonbein, G. W. (1999). Biomechanics of microcirculatory blood perfusion. Ann. Rev. Biomed. Eng. 1, 73-102.

Secomb, T. W. and R. Hsu (1996). Motion of red blood cells in capillaries with variable cross-sections. J. Biomech. Eng. 118, 538-544.

Sholley, M. M., G. P. Ferguson, H. R. Seibel, J. L. Montour and J. D. Wilson (1984). Mechanisms of neovascularization. Vascular sprouting can occur without proliferation of endothelial cells. Lab. Invest. 51, 624-634.

Stokes, C. L., M. A. Rupnick, S. K. Williams and D. A. Lauffenburger (1990). Chemotaxis of human microvessel endothelial cells in response to acidic fibroblast growth factor. Lab. Invest. 63, 657-668.

Stokes, C. L. and D. A. Lauffenburger (1991). Analysis of the roles of microvessel endothelial cell random motility and chemotaxis in angiogenesis. J. Theor. Biol. 152, 377-403.

Sutton, D. W. and G. W. Schmid-Schonbein (1994). The influence of pure erythrocyte suspensions on the pressure-flow relation in rat skeletal muscle. Biorheology 32, 107-120.

Terranova, V. P., R. Diflorio, R. M. Lyall, S. Hic, R. Friesel and T. Maciag (1985). Human endothelial cells are chemotactic to endothelial cell growth factor and heparin. J. Cell Biol. 101, 2330-2334.

Warren, B. A. (1966). The growth of the blood supply to melanoma transplants in the hamster cheek pouch. Lab. Invest. 15, 464-473.

Wilkinson, D. and J. F. Willemsen (1983). Invasion percolation—a new form of percolation. J. Phys. A. 16, 3365.

Williams, S. K. (1987). Isolation and culture of microvessel and large-vessel endothelial cells; their use in transport and clinical studies, in Microvascular Perfusion and Transport in Health and Disease, P. McDonagh (Ed.), Basel: Karger, pp. 204-245. 